\title{
The Primary Pitfalls on the Road to Understanding Consciousness
}

\author{
Karl Sipfle
}

Independently, at NASA GSFC

ksipfle@umich.edu

November 18, 2020

\begin{abstract}
Consciousness and the human mind have been pondered for centuries. Today the most fundamental, likely outlines of the explanation of consciousness are known, and from this landscape we are wellpositioned to fill in the basket of specific theories that will include the correct one. However, many errors in thinking and assumptions have accumulated, and even gained fashion, that obscure our progress. It is time to eliminate these, which we do herein by enumerating the complementary true assertions. Following that we mention four principles for choosing direction for further work. We conclude with a framework for further progress.
\end{abstract}

\section{Contents}

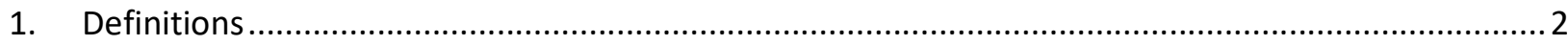

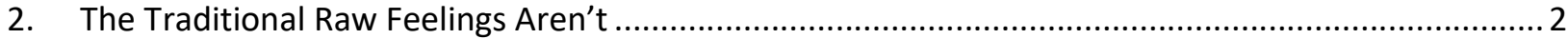

3. That Which We Can See at the Bottom of Our Own Minds from Within is Not the Actual Bottom .... 3

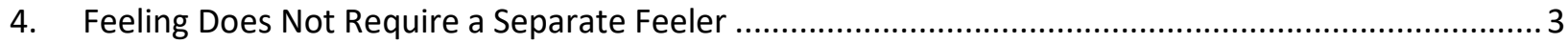

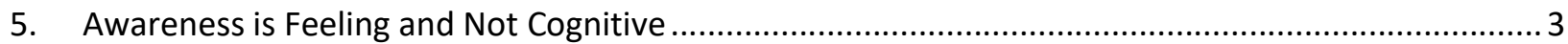

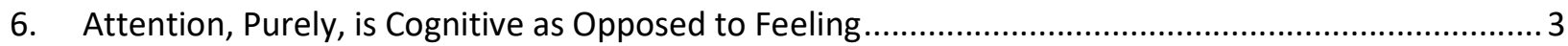

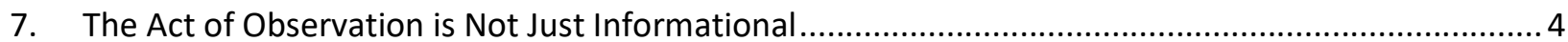

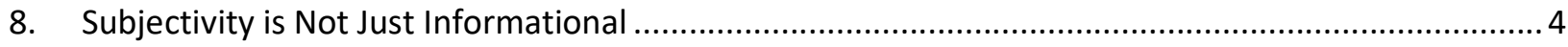

9. Consciousness is an Epiphenomenon and Consciousness is Not an Epiphenomenon .......................4

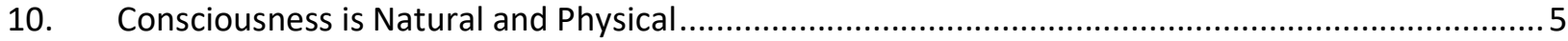

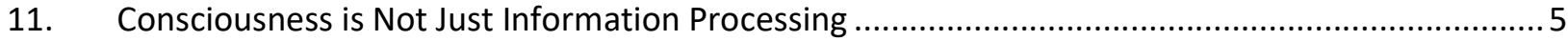

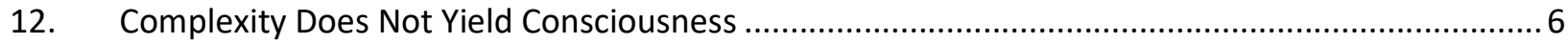

13. Mind is Not Unitary Except Through Fusion of Constituent Parts ............................................. 6

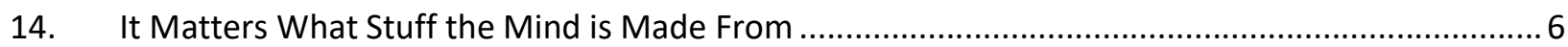

15. The Building of the Machinery for Human Cognition Required and Requires Feeling ................... 7

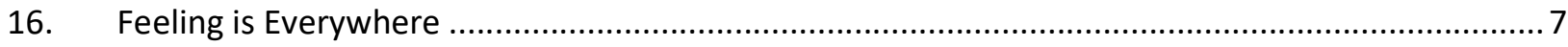

17. There is No Conflict Between Free Will and Mechanism.........................................................

18. Procedural: Incrementalism Plus Empiricism Will Not Discover the Answer First ........................ 7 
19. Procedural: Soft Laws are Valuable

20. Procedural: Excess Aversion to Addition is Counterproductive ................................................ 8

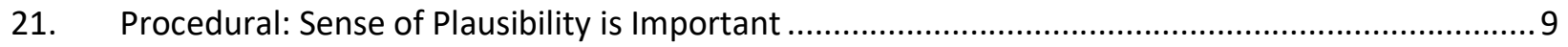

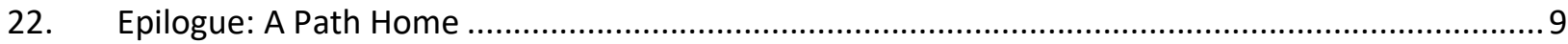

References

Error! Bookmark not defined.

\section{Definitions}

In few areas of inquiry are definitions more important than the study of consciousness. Our choices below try to best accommodate common shades of meaning.

Consciousness is a bubble of experience (Gamez, 2018). It is something found in and recognizable by a mind.

Fundamental consciousness aka Pre-consciousness is the smallest possible particle of experience.

Feeling is what is commonly called subjective experience, and the central concern of the Hard Problem.

Raw feels are the simplest feelings.

Qualia are alleged raw feels. Some qualia are micro-qualia or collections of identical micro-qualia.

Micro-qualia are true, indivisible raw feels.

Cognition is thought and not feeling- information processing. In practice feelings may accompany cognition.

\section{The Traditional Raw Feelings Aren't}

Qualia are discovered by the introspection of a mind. These are the experiences that specific minds report as simplest units of experience that they are able to discern. A tragic leap has been made from the simplest to a mind, to the simplest of all- that is, to the universe that has forged the mind. This is a massive and incorrect assumption.

The traditional qualia such as the taste of a wine are not fundamental feels. The experience of a headache is not a fundamental feel. However, Pain (and likewise Pleasure), unqualified and unelaborated pure dysphoria, are fundamentals. They are micro-qualia, and (in sufficient synchronized numbers to emerge in a mind) are also qualia.

How do we know pain and pleasure are fundamental? Because you cannot make "hot," valent feeling out of "cold," neutral components, no matter how assembled. As with atoms, neutral and distinguishable assemblies can be made from the valent, but not vice versa. Even if a neutral item can 
be split into a positive and negative pair, this would a) have to be on the scale of base units to yield significant net valence, and b) yield what is fundamental beneath conscious minds- valent feeling.

Information is capture of pattern and can encode valence but is not itself valent. Valent items can, however, be arranged into informational patterns.

\section{That Which We Can See at the Bottom of Our Own Minds from Within is Not the Actual Bottom}

We can look down from inside and we can look up from physics. There are two vast regions to be charted. The first is the gap between physics and the lowest things we can see from inside- this span is not of zero size, there is amalgamation and elaboration. The second is all the structure between the lowest things we can internally perceive and the full-blown thoughts and emotions that are the normal coin of our fully conscious life.

\section{Feeling Does Not Require a Separate Feeler}

Because we exist of our own nature and at our large scale, people have assumed that- even for "one's own feelings"- there must be an experiencer of "those" feelings. At the bottom, feeling happens. (Sipfle, 2018). It exists freestanding. It does not happen to something else, at least no further than in the sense of particles mutually interacting in and as the fabric of the universe. This is how the descending homunculi disappear.

Cognition rests on even less. With pure feeling, there is no pattern and the nature of whatever is happening is everything. With information, it matters not what the stuff is that is arranged into patterns, only how it is arranged. This means that with a full reduction the only stuff that is needed is the feeling stuff!

\section{Awareness is Feeling and Not Cognitive}

You can feel the presence of something before and without any thinking about it and without even knowing what or where it is. In contrast, a mechanism (including in a brain) can be "aware" of something and respond to it, but it is not doing what we do when we say we are aware of something. The difference is feeling. The same responsive acts performed many times become rituals and fade from awareness.

\section{Attention, Purely, is Cognitive as Opposed to Feeling}

Attention is selection of what to focus on. It allows a single difficult-to-evolve unit to process one (or a few) at a time of many inputs, and thereby serve all the inputs. Attention may use feeling as a selection determiner and operate on feelings as the things selected, but mechanism of switching between inputs 
(attending) is mechanical ("cold") itself. One can, however, be additionally aware of what one is attending to, including both one's own thoughts and one's own feelings.

\section{The Act of Observation is Not Just Informational}

To observe includes to become aware of and become aware of related detail.

\section{Subjectivity is Not Just Informational}

The realm of the "subjective" includes both "subjective experience" and "subjectivity."

Subjective experience is not informational, it is the opposite of that: feeling.

Our Subjectivity has been described as an informational relativity. At a very high level and with discipline (which is feeling!) a mind can be trained to consider formal things where this would be so. But that is not at the core of what Subjectivity actually is to a brain and how it works.

A "Subject" is born when a clot of feelings is affected by something outside itself- when, at a higher than fundamental level there is now a distinction between the experiencer and the experienced. When the other something is in the same mind, we have what amounts to one clot of feelings experiencing another clot of feelings. (This can result in bad feelings regenerating further bad feelings mushrooming into a widespread dysphoria.)

In practice most such subjective events for us are further elaborated both at the experiencing site and the experienced site with informational content, giving rise to complex emotions as well as feeling-laden observations and introspections.

\section{Consciousness is an Epiphenomenon and Consciousness is Not an Epiphenomenon}

The Hard Problem (Chalmers, 1995) itself suggests that pain and pleasure 1) serve a purpose that 2) might have been served by some other mechanism in some other universe. They key is to provide valent value serving to steer judgements (at all levels and scales).

The question becomes epi- in what way, epi- to what exactly? The subjective experience Is not an epiphenomenon in the sense that it provides the valent that is used by animal brains to steer cognitive circuits and select and enhance memories. It is an epiphenomenon in that, if there is something that can do the same job in some other universe, that other phenomenon would not have to be feeling.

Circuits can first be steered by this conscious experience and then be written into nonconscious elements once correctly shaped. We see this sort of mechanism when we learn to drive a car and it also happens in evolutionary time periods affecting wiring. 


\section{Consciousness is Natural and Physical}

Consciousness occurs in our universe; it is natural. And, though crudely, minds are able to interact successfully by way of beings of and operating in the physical world.

Every important and mysterious phenomenon we experience has fallen to a physics description save this one. There is every reason to expect this one will, too, and little reason to doubt that. In the universe in which we find ourselves, we find a describable order in all things and happenings.

To be sure, consciousness is a strange thing, but so were many other now-accepted concepts of science, such as action at a distance and electromagnetic waves and quantum behaviors.

If we bring an electron near another one, the first will scoot away. That would be very strange to encounter for the first time, yet at least that case resembles in some ways our familiar world of macroscale objects.

Since then we have found quantum behaviors that to our minds crafted for the Newtonian macroscale have been hard to understand, but we have found the many correlations to objects we are familiar with and so have become convinced.

Thus, we have been here before. We are so early in our search for consciousness that there is much less to attach to; with a few more puzzle pieces in place it will become easier.

Feeling is a physical phenomenon that we can expect to be explained exactly as far as the rest. Why does the electron move? We can say "because of a force" but that just defers the query by one step. When we reach the fundamental, the answer is "it just does," which is fine because what that really means is "because that is a part of a system which we know is possible because it exists, and because this particular possible system can support inquirers like ourselves."

We are even more "inside" consciousness than gravitation, which makes the big picture harder to see, but we will have equivalent realizations to that of us being at one star inside a galaxy among many galaxies.

A certain particle interaction emits a neutrino. Why? Within certain constraints, it just does. So, too, with feeling. When we find the link to the movements in our brains, we will be comfortable with the new facts.

We expect that minds are built of very small things and not born naïve but whole.

\section{Consciousness is Not Just Information Processing}

Consciousness requires both cognition and feeling. While one can't make pain or pleasure from neutral objects, even less can one make them from nothing at all (information). Neither the pattern nor cold pebbles placed to represent it (no matter how complex the arrangement) can make feeling.

The cognitive is $100 \%$ equivalent to a simulation; feeling is not.

The human mind is not equivalent to a simulation for one reason and that is because it actually feels. Here we see the difference between an abstraction like mathematics, which can carry information, 
which is itself simply description of pattern of something, and the actual. Consciousness requires the actual.

\section{Complexity Does Not Yield Consciousness}

There is a body of argument extant that posits that consciousness appears because brains (or artificial devices) eventually become complex enough. Though as for any machine there will need to be a certain complexity, this is not the source of consciousness.

The position misunderstands systems theory. Systems can indeed exhibit behaviors that one would never have predicted by just looking at the constituent parts (though they can be discovered by simulations incorporating models of the parts), but that does not mean a system can exhibit any phenomenon if only we arrange its parts with enough complexity.

You can arrange ball bearings all day long and you will never be able to make a living, feeling, sheep out of them. It matters what the parts are, what their nature is, what their potential is insofar as what is possible to make from them.

If feeling should suddenly appear from complexity, then a) we will have discovered not an "explanation" but a new brute fact of the universe, like electric charge, and b) this will very probably most minimally appear at a very small (molecular scale at most) level and with a simple arrangement. In other words, it will be a simple fundamental, not a complexity result!

Like everything else, the consciousness we know arose from something already there of the same basic nature.

\section{Mind is Not Unitary Except Through Fusion of Constituent}

\section{Parts}

The illusion of the mind as a unitary object tends to bias our thinking unhelpfully. Yet, this fusion of experience is also a key clue into how the mind works and especially as to how consciousness works in it.

\section{It Matters What Stuff the Mind is Made From}

Our minds cannot be made or remade of arbitrary materials and mechanisms. There are multiple ways the computation could have been implemented because the material is just placeholders to represent information (although most of these ways ultimately rely on electromagnetism and matter).

But feeling requires that whatever physical mechanism is at play is represented in the brain, by constituents that play that way. 


\section{The Building of the Machinery for Human Cognition Required and Requires Feeling}

While cognition is not about feeling, the mill that performs cognition uses feeling in its operation and in evolutionary bootstrapping toward it.

Feeling has immediate effect in directing suitable flows of information, which may in later stages of evolution be captured in circuits that no longer need to feel. In this case the subjects that are then associated with good or bad feelings are higher-level than were those of earlier times.

\section{Feeling is Everywhere}

Animals are organized constructions that harness a few basic behaviors of the universe to enable animal-level actions, promoting competitive genome survival. Minds and what they do are just one more example of this. Minds necessarily had to be formed from what was, in disorganized form, already present.

\section{There is No Conflict Between Free Will and Mechanism}

At times hand-wringing over Free Will has entered the search for consciousness. One does what one Wants to do, all things considered, based on present and anticipated pains and pleasures. This can be stated either as a definition or an observation and is true in both cases. In fact, how your brain reacts to all the various pains and pleasures in making its decisions is what your personality is (Rolls, 2016). Your Will is what you want. Like all other bodily functions, it is a fact of nature.

"Free" is a relativistic term. One may be free from other people's control. As a creature of the universe you are not free of its control. It also enables and implements you.

\section{Procedural: Incrementalism Plus Empiricism Will Not}

\section{Discover the Answer First}

Next, we move to cautions about procedure rather than natural truth.

The correct answer for consciousness will come originally from advanced imagination and suspicion, to be later confirmed by experimentalists. In particular, the notion that if one cannot now detect something with the instruments we now know how to make then it probably doesn't exist, is woefully impotent today on the topic of consciousness. "Laws" (including conservations) have been broken on the way to the Standard Model; without being willing to consider these possibilities we would not have reached our successful Model. Consciousness is stranger still than earlier challenges and leaps are necessary; compiling a compelling body of evidence experimentally to demand one answer would take a very long time, measured, as one wag put it, in the unit of dead scientists. 
Consciousness takes us into BSM (Beyond the Standard Model) physics- we know this from the start.

\section{Procedural: Soft Laws are Valuable}

It is not certain that the laws of consciousness will much resemble the laws of the other physical phenomena; there is no logical proof of this and there is no empirical proof today, either. However, two things push us to that plausibility, and both are based on general experience only. The first is Occam's Razor; we have found that the correct answers tend strongly to be among the simplest possible answers. This is one manifestation of a second general and true rule of our universe that appears ubiquitously, which is that that which has been found to be the case many times will probably appear repeatedly again.

\section{Procedural: Excess Aversion to Addition is Counterproductive} It is proper (wise) to minimize in a new theory the bringing into being of new objects and concepts with little empirical evidence to back them up (an ontological aversion).

However, it is a mistake to meticulously avoid this when all other avenues are yet more contrived and arbitrary and implausible. The bar should be high, but not infinitely so.

A true Theory of All (TOA) will be Standard Model + Gravity + Consciousness. There must be enough conceptual material to cover that span.

As with consciousness we have other mysteries such as dark energy and dark matter, and ordinary matter and gravitation. As the later pieces of the physics puzzle are filled in, multiple mysteries may fall at the same stroke. Consciousness may be related to or even identical to one or more of these. Consciousness may turn out to be not only a universal stuff but the universal stuff, an inversion of our prior thought paths.

With no more actually required than the observation that all other forces have messenger particles scientists have been justified speculating on a graviton. Similarly, an inflaton to explain cosmic inflation. Einstein's contributions were often arguments of logic made from a small number of basic empirical facts; his mathematics of gravitation were one possibility only later upheld empirically.

Metatheories- that is, bounded collections of theories- are a sensible vehicle on the journey, and these may even be neutral on questions re new objects. A logical course, for example, is to point to and name a new particle, with the explicit proviso that it may actually be new, or a familiar particle used by Nature in a previously unknown way (like electromagnetics being married to electrostatics).

It is not only possible, but likely, that the physical description of consciousness will involve both familiar and strange structure. 


\section{Procedural: Sense of Plausibility is Important}

While there is little to go on, speculation is necessary and skill in recognizing the plausible (especially where change is required) increases in value.

Consciousness theories exist featuring no discussion on why it may be a likely true or nearly true theory or metatheory. Many things could be true- which are? This is a hard type of evidence to express (and it is evidence)- it relies on intuition and judgement not necessarily felt by the shaped neural nets of others, but it is precisely these kinds of leaps that first speculate the correct paradigm shifts (this we call "genius"). Timidity does not serve at the frontier.

\section{Epilogue: A Path Home}

How do we solve consciousness? High enough analysis and in the right way, "lower than philosophy, higher than our standard physics."

We have physics laid down now that mostly helps us but also tricks us by asserting patterns that may not entirely hold for something as strange as consciousness. One excellent clue from physics that probably does hold, is that everything fundamental about consciousness probably does not require large expanses of space or time.

The first step is to realize that feeling is an action, and apparently a fundamental action. Given that it is a fundamental action that is not the same as the others, as is the way of our physics we posit a field for it as well. It follows that there is also a charge and at least one associated force particle.

We frame this as a metatheory, admitting that we don't yet know the specifics of this field, nor its relation to the existing ones. We acknowledge that "new" particles could be existing particles about which we have incomplete knowledge. Nonetheless the first logical position is to frame the theory in terms of consciousness without commitment on these finer points either way. This is not falsity, it is generality.

Until we are able to do empirical experiments that are shown to have access to consciousness, we must rely on informed speculation with an emphasis on plausibility, and thought experiment, and avoidance of the easy pitfalls discussed.

We must recognize that it is far from certain that the laws of consciousness respect, for example, special relativity, or operate mostly in our familiar dimensions of space and time.

We should maintain the obvious suspicion that consciousness may have something to do with- perhaps a great deal to do with- collapse of the wave function or gravitation or dark energy or matter as we know it.

We must entertain theories that have less empirical support than we have been comfortable with. We must recognize that while most imaginings are false, a few are true. We must mine our sense of the plausible because our understanding of the truly required is unable to serve us sufficiently here.

We must create metatheory and catalog the metatheories and see how precisely we can describe and bound them, despite the risk that entails. 
We must appreciate complexity layering from fields and particles up to animal minds and come to see how these happen.

The degree of explanation of the forces we know is nothing more than a descriptive catalog of which particle combinations interact and how strongly. In the absence of any strong evidence to the contrary we should assume the same posture with consciousness.

What we know so far suggests that consciousness is a) pervasive, b) very fundamental

and c) that we don't just have it, we are made out of it- how much so is an intriguing question.

How to prove is an important but separate problem from figuring out a plausible approximate explanatory theory or class of theories (metatheory). While we are unfortunately missing means to test, we should not idle ourselves in waiting, and likely the very hard brain work of the present era will suggest the experiments. Many of the really important items we have found were not discovered until after we developed better provisional ideas of what to look for.

There are interesting precedents as humankind realizes and proves (in that order) how ever more deeply we are made of that which surrounds us:

We are animals, too.

We are made of cells like all other life here.

We are made mostly of the water that everywhere surrounds us, and emerged from it.

Our Sun is one of the stars.

We and the Sun are made of older stars.

Now consciousness we understand is an energy of the universe and not somehow just found in animal heads. Now we find ourselves in a universe laden with dark matter and infused with dark energy as our minds ponder what "other" things these are, and what feelings are, and how exactly probabilities and desires become actualities. It will be strange indeed if we do not find yet deeper kinship in nature.

\section{References}

Chalmers, D. (1995). Facing up to the problem of consciousness. Journal of Consciousness Studies, 2: 200-19.

Gamez. (2018). Human and Machine Counsciousness. Cambridge, UK: Open Book Publishers.

Rolls, E. T. (2016). Cerebral Cortex: Principles of Operation. New York: Oxford University Press.

Sipfle, K. (2018). The Nature of Consciousness. New York: Barnes and Noble Press. 
